Artery Syndrome, when their semeiology is complete, beyond doubt, are a supplementary anatomical and functional precision.

The description of a spinal cord or root injury can be only exhaustive if it is accompanied by the statement of flaccidity or spasticity, and, furthermore, a description of the remaining functional possibilities.

We approve, without reserve, the effort made by Michaelis in his attempt to clarify and unite the 'paraplegist's language'.

It would, nevertheless, be a great achievement to agree, on the territory of each segment: myotome, dermatome, as well as the bony, articular and visceral one.

We have thought indispensable to put forward the advantages of the French method, which is, at the same time, simple and logical.

Chairman. A 'battle' between the French and the Anglo-Saxons seems to have started. Of course, I can see already it will be difficult to develop a Common Market in the nomenclature, but we have to try our best to do it. I personally have a slight doubt about that remark of the more exact expression in the French language. I think every other language can make the same claim.

\title{
METAMERICAL TOPOGRAPHY OF THE CORD AND ITS ROOTS
}

\author{
By Jean Benassy, M.D. \\ Hôpital Raymond-Poincarè, Garches
}

THE study of the para- and tetraplegic patients allows us not to establish the different metameric levels of the cord and of its roots, and we hope that everybody will agree with the following propositions.

\section{UPPER LIMB}

C5 Root (fig. I). In its area of supply are found deltoideus, supra-spinatus, infra-spinatus, biceps, brachialis and supinators. The function of $\mathrm{C}_{5}$ is abduction of the arm, external rotation of the shoulder, flexion and supination of the forearm. It is the position of the arm which is usually adopted by tetraplegics with a complete lesion below $\mathrm{C}_{5}$, at least during the acute stage-X-ray shows the scapula fixed parallel to the vertebral column as a result of the action of the rhomboids. The deltoideus and the supra-spinatus drag the humerus laterally, the subscapularis being extended passively. The topography of the muscles innervated by this root is characteristic. Its long muscular bulk extends from the upper part of the limb, clavicular and spine of the scapula to the tip of the styloid apophysis of the radius constituting an homogeneous muscular group.

In this chart we do not include serratus anterior; it is an easy muscle to test. Occasionally paralysis of this muscle occurs as a result of an accidental section of the nerve of Charles Bell in Halstedt's operation. However, in our experience of patients suffering from brachial plexus lesions or in tetraplegics, we have never seen a paralysis of the serratus anterior. If, macroscopically, the nerve of Charles Bell appears to originate from the brachial plexus, it appears that the fibres which innervate serratus anterior come from the anastomosis between the fourth and fifth cervical roots and thus from the cervical plexus. This we regard as important, since the serratus is an accessory external inspirator muscle, as the diaphragm. It 
follows that $C_{4}$ is essentially an inspiratory root controlling the two principal inspiratory muscles, namely the diaphragm and serratus anterior.

C6 Root (fig. 2). C6 innervates three large muscles which have the common function of adduction of the arm: latissimus dorsi, teres major and pectoralis major. In fact, only the upper head of pectoralis major is innervated by C6. It also includes

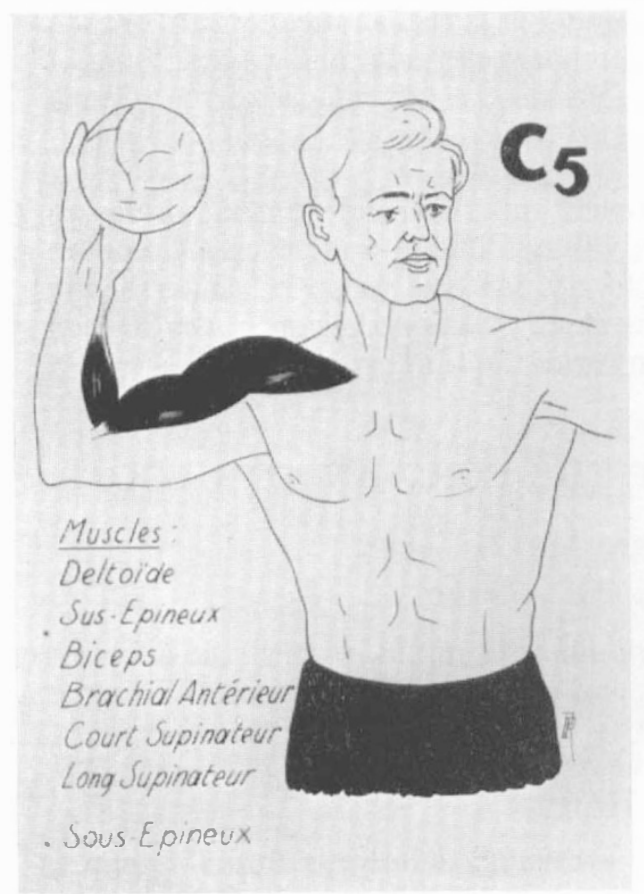

FIG. I

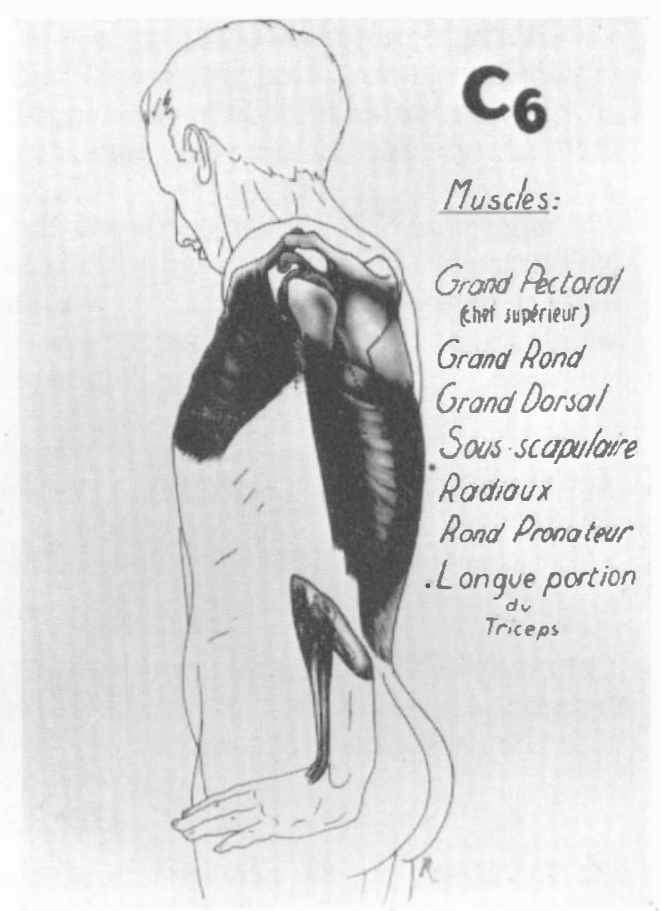

FIG. 2

TABLE I

Segmental Motor Innervation of the Upper Limb

C5 Deltoideus

Supra-spinatus

Infra-spinatus

$\mathrm{Bi}$ : 冫⿰氵工力s

Brachialis

Supinator longus

Supinator Brevis

C6 Teres major

Latissimus dorsi

Pectoralis major (clavicular head)

Sub-scapularis

Triceps brachii (long head)

Ext. Carpi Radiales

Pronator teres
$\mathrm{C}_{7}$ Triceps Brachii (Vasti)

Flex. carpi radialis

Ext. digitorum comm.

Ext. pollicis longus

C8 Flex. carpi Ulnaris

Flex. digitorum sublimis

Lumbricales

Flex. digitorum prof. comm.

Flex. Pollicis prop.

TI Interossei

Pectoralis major (Sternal heads)

Opponens pollicis 
the subscapularis which inwardly rotates the shoulder; furthermore, two others which have a very different function, the pronator teres and the extensor of the wrist.

Its morphology therefore is very different from that of $\mathrm{C}_{5}$. The three big muscles form a group which extends over the thorax issuing from the sternum, the costae and the inner two-thirds of the clavicula to lie as a single tendon in the sulcus at the anterior part of the head of the humerus, which acts as a bony relay. From there proceed teres major and latissimus dorsi to insert on the edge of the scapula, the spine and the posterior two-thirds of the iliac crest. This takes the form of butterfly's wings, and if we consider these muscles on both sides we see that they embrace the thorax from the sternocostal angle to the ilio-vertebral angle in a direction which is at a right angle to the general axis of the costae.

The physiology of the adductor group of the upper limb innervation by C6 is of very great interest as auxiliary expiratory muscles.

Tetraplegics with lesions above C6 cannot laugh, sing or whistle. If they attempt to laugh, they produce a form of inspiratory movement. These patients have very serious respiratory impairments; but if the lesion is below the level of C6 they recover a nearly normal respiration. During the act of laughing, in pectoralis major, latissimus dorsi and teres major, powerful contractions can be felt in the axilla. While the fourth cervical root may be regarded as inspiratory, C6 may be regarded as expiratory, on support of the abdominal muscles.

\section{LOWER LIMB}

LI Root (fig. 3). It innervates the sartorius. Either temporarily or permanently a large number of paraplegics have only functional use of this muscle, which must be placed highest of all muscles in the segmental innervation of the lower limb.

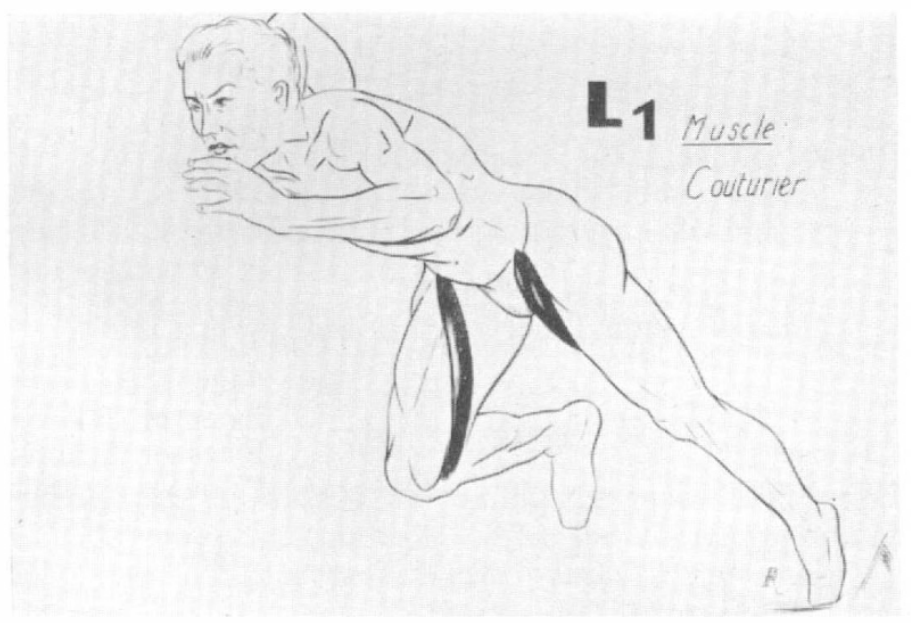

FIG. 3

This name of sartorius is a physiological misname; 'Starter' would be possibly better; in fact, we must remember that the root LI gives always way to a big white 
ramus, conducting some genital sensations and the pathways of the sphincter of verumontanum.

The sensibility of the testicle is of the LI level.

L2 Root. This root innervates the iliopsoas, the rectus anterior and the pectineus. Both rectus anterior and iliopsoas, are flexors of the hip. The upper adductors have the same high innervation.

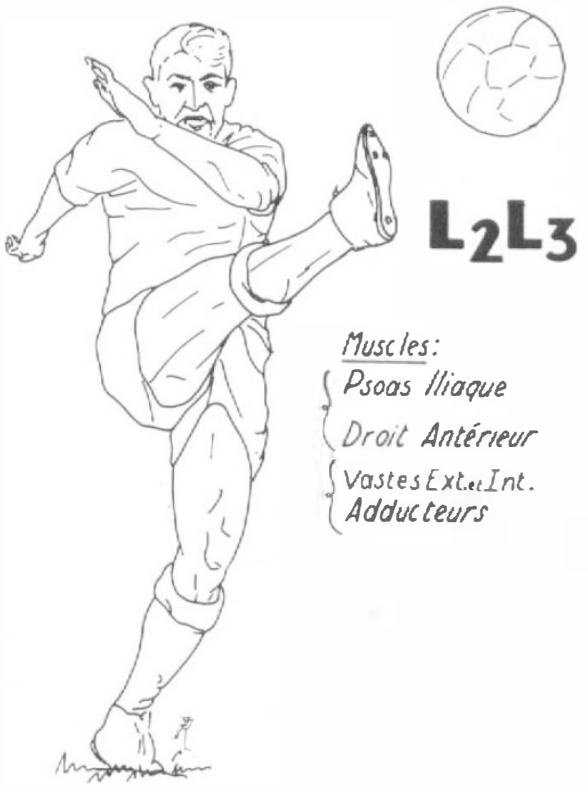

Fig. 4

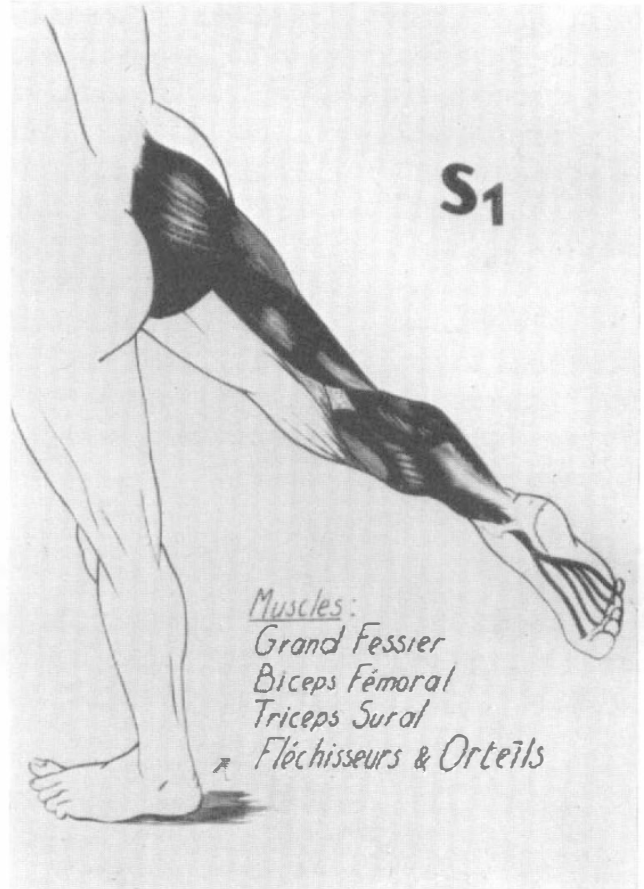

FIG. 5

TABLE II

Segmental Motor Innervation of the Lower Limb

LI Sartorius

L2 Psoas iliacus

Rectus femoris

Pectineus

L3 Gracilis

Vasti (medialis and lateralis)

L4 Inner hamstrings

Tibialis anticus

Tibialis posticus
L5 Gluteus medius

Extensor Hallucis longus

Extensor digitorum comm.

Peroneus Lateralis

SI Gluteus medius

Biceps femoris

Triceps surae

Flexor hallucis prop.

Flexor digitorum comm.

S2 Small plantar muscles 
L3 Root (fig. 4). It controls the vasti medialis and lateralis and the gracilis which is an adductor and flexor of the leg. Grossiord rightly draws attention to the importance of this muscle, which is not normally well developed, but which, after proper re-education, can play an important part in the rehabilitation of the polio or paraplegic patient. It is of great importance to test this muscle accurately and differentiate it from the hamstrings.

In observing the natural kick of a footballer, one realises that both $\mathrm{L}_{2}$ and $\mathrm{L}_{3}$ roots are fully involved, which means that the kick is directed inwardly and, on the other hand, the outward kick of a ball would be only achieved with difficulty.

L4 Root. In our opinion it innervates both the tibialis anticus and posticus. Following operations on L5 disc, it is interesting to test the patients the same evening; one can then notice a slight weakness of the extensor digitorum but will always find that the tibialis anticus responds normally. Opinions differ in regard to the tibialis posticus; it is known as the 'sailor's muscle', since it is the tibialis posticus which allows him to climb masts.

In the majority of cases it also had its innervation in $\mathrm{L}_{4}$.

The two muscles seem to have opposing actions since one dorsiflexes the foot and that the other acts as an plantar flexor. In fact, their most important function is the adduction of the foot. The L4 root controls supination of the foot.

L5 Root. It supplies the following muscles: gluteus medius, extensors of the toes, peroneus lateralis. It is responsible for abduction of the hip, pronation of the foot and extension of the toes.

Si Root (fig. 5). It supplies a very large area which includes gluteus maximus, biceps femoris, gastrocnemius and flexor digitorum. Those muscles which seem to have different action, are nevertheless synergical.

In running, the back-kick of the leg is controlled by the first sacral root. The forward action of the leg is performed by $\mathrm{L}_{2}-\mathrm{L}_{3}$. Roots $\mathrm{L}_{4}$ and $\mathrm{L}_{5}$ function as lateral stabilisers.

S2 Root. This root seems to perform a function similar in the foot as does $\mathrm{TI}_{\mathrm{I}}$ in the hand. Its distribution supplies the small plantar muscles. The semiological action of this root is important since its paralysis results in a clawing of the toes, comparable to the claw of the fingers.

As $\mathrm{S}_{2}$ gives innervation to the anterior portion of the perineal muscles, this clawing of the toes often indicates severe impairment in the function of the bladder.

\section{CONCLUSION}

Until recently our knowledge concerning the motor segmental innervation of the limbs was somewhat confused. The french neurophysiologist Morin recently claimed that 'the metamerical distribution of the anterior roots has no functional character', and maintained that Paul Bert who contended that the same root innervates only synergical muscles was under a misapprehension. Our own experience does not lead us to agree with this, and accepting rather Paul Bert's views, we believe that there is a remarkable systematisation in the functional distribution of the motor roots. 